\title{
Diagnóstico dos serviços de saneamento urbano do município de Porto Nacional/TO
}

O objetivo deste trabalho foi analisar a evolução do atendimento dos serviços públicos de abastecimento de água e de esgotamento sanitário no município de Porto Nacional - TO, com base nos dados Sistema Nacional de Informações sobre Saneamento Básico (SNIS). Os dados utilizados nesta etapa foram obtidos no sítio eletrônico do SNIS, referentes ao município de Porto Nacional, no período compreendido entre os anos de 2011 e 2019, a análise histórica de atendimento consistiu na comparação dos percentuais de prestação dos indicadores do ano de 2018 com o percentual de anos anteriores. As metas de curto e médio prazo previstas no Plansab para a região Norte foram superadas e os indicadores se mantem próximos das metas previstas para o Brasil.

Palavras-chave: Saneamento básico; Indicadores; Universalização.

\section{Diagnosis of urban sanitation services in the municipality of Porto Nacional/TO}

The objective of this work was to analyze the evolution of the attendance of public water supply and sewage services in the municipality of Porto Nacional - TO based on data from the National Basic Sanitation Information System (SNIS). The data used in this stage were obtained on the SNIS website, referring to the municipality of Porto Nacional, in the period between the years 2011 and 2019, the historical analysis of service consisted in comparing the percentages of provision of the indicators of the year $\mathbf{2 0 1 8}$ with the percentage of previous years. The short and medium-term goals foreseen in the Plansab for the North region have been exceeded and the indicators remain close to those foreseen for Brazil.

Keywords: Sanitation; Indicators; Universalization

Topic: Engenharia de Recursos Hídricos

Reviewed anonymously in the process of blind peer
Received: $\mathbf{1 0 / 1 1 / 2 0 2 0}$

Approved: 15/02/2021
Jackelliny Cristina Neves do Nascimento Instituto tocantinense Antônio Carlos, Brasil http://lattes.cnpq.br/0829739633905211

jackelliny-cristina@bol.com.br

Alesi Teixeira Mendes

ITPAC Porto Nacional, Brasil

http://lattes.cnpq.br/2473113080999082

http://orcid.org/0000-0002-5632-7235

alesi.mendes@itpacporto.edu.br

\section{Referencing this:}

NASCIMENTO, J. C. N.; MENDES, A. T.. Diagnóstico dos serviços de saneamento urbano do município de Porto Nacional/TO. Natural Resources, v.11, n.1, p.41-47, 2021. DOI:

http://doi.org/10.6008/CBPC2237-9290.2021.001.0006 


\section{INTRODUÇÃO}

Em todo o mundo, a água e o saneamento são reconhecidos como direitos humanos, mas ainda há muito a ser feito rumo à universalização dos serviços de abastecimento de água, coleta e tratamento de esgoto, coleta e destinação adequada de resíduos sólidos e drenagem urbana. A falta de saneamento é degradante, desagradável, insalubre e prevalente, mesmo em áreas urbanas.

De acordo com o Ranking de Universalização do Saneamento 2018 da Associação Brasileira de Engenharia Sanitária e Ambiental (ABES, 2018), a maioria dos municípios brasileiros está empenhada na universalização do saneamento, porém um percentual pequeno $(10 \%)$ tem de fato demonstrado compromisso com a universalização e menos de $5 \%$ alcançaram o objetivo.

$\mathrm{Na}$ eminência de alcançar o horizonte de médio prazo do Plansab para a universalização dos serviços (2023), a realidade do Brasil evidencia a distância entre os indicadores atuais de atendimento e as metas propostas. De acordo com o Plano Nacional, em 2023, o Brasil deve contar com 95\% de domicílios atendidos com abastecimento de água e segundo os dados do Sistema Nacional de Informações sobre Saneamento (SNIS), em 2018, 83\% da população tem acesso a esse serviço. O cenário mais preocupante envolve o esgotamento sanitário, enquanto a meta prevista estabelece que $81 \%$ de domicílios estejam servidos com coleta de esgotos sanitários, apenas 53\% da população brasileira é atendida (BRASIL, 2014).

Diante desse quadro nacional a realidade do norte do país é ainda mais alarmante. Uma análise agrupada dos Estados por regiões torna mais evidente quais parcelas do país estão mais distantes do ideal da universalização. Tratando-se de acesso a água potável, os Estados das regiões Sudeste e Sul do Brasil possuem, respectivamente, $91,03 \%$ e $90,19 \%$ de atendimento, seguidos do Centro-oeste com $88,98 \%$ e do Nordeste com $74,21 \%$, e em último lugar a região Norte com $57,05 \%$ da população abastecida com água tratada. Em matéria de esgotamento sanitário, o Norte do país fica mais uma vez em último lugar com 10,49\% de atendimento, quando comparado aos $79,21 \%$ da região Sudeste fica evidente a disparidade regional que ainda existe no Brasil quanto a universalização dos serviços de saneamento básico (BRASIL, 2018).

Diante disso, este trabalho foi desenvolvido com o objetivo analisar a evolução do atendimento dos serviços públicos de abastecimento de água e de esgotamento sanitário no município de Porto Nacional TO, com base nos dados Sistema Nacional de Informações sobre Saneamento Básico (SNIS).

\section{REVISÃO TEÓRICA}

\section{Bases de dados sobre saneamento básico disponíveis no Brasil}

As dimensões continentais do Brasil e a diferença, em diferentes termos (geográfica, econômica, institucional etc.) dos seus municípios torna complexa a tarefa de estabelecer uma base de dados nacional que seja realista, transparente e que alcance a totalidade dessas localidades. No entanto, o país conta com bases de dados disponíveis relativas ao saneamento sob responsabilidades de diferentes órgãos e entidades, a nível de exemplo se pode citar: 1) o SNIS do Ministério do Desenvolvimento Regional; 2) Censo demográfico e Pesquisa Nacional por Amostra de Domicílio (PNAD) e a 3) Pesquisa Nacional de Saneamento Básico (PNSB) 
do Instituto Brasileiro de Geografia e Estatística (IBGE); e os 4) Atlas Brasil: Abastecimento Urbano de Água e 5) Atlas Esgoto: Despoluição de Bacias Hidrográficas da Agência Nacional de Águas e Saneamento Básico (ANA).

O SNIS é mais importante sistema de informações do setor de saneamento do Brasil, criado em 1996 e vinculado à Secretaria Nacional de Saneamento Ambiental, do Ministério das Cidades. O SNIS é um banco de dados cuja administração é federal e contém informações sobre a prestação de serviços de água, esgoto, resíduos sólidos e águas pluviais. Atualmente, o sistema é vinculado à Secretaria Nacional de Saneamento do Ministério do Desenvolvimento Regional.

Os indicadores do SNIS são distribuídos nos seguintes grupos: Atendimento, Operacionais, Econômicos, Financeiros e Administrativos, Investimento total por ligação de água e esgoto, Investimento Total por habitante, Investimentos realizados com Recursos Onerosos, Investimentos realizados com Recursos não Onerosos e Investimentos realizados com Recursos Próprios e um Indicador de Qualidade (SCRIPTORE et al., 2012).

Os dados do SNIS são coletados anualmente em um processo auto declaratório por parte dos municípios e prestadores de serviços de saneamento básico, seguindo o cronograma de coleta de cada componente. Em vistas desse caráter autodeclarado dos dados fornecidos pelos prestadores de serviço faz com que não se tenha certeza acerca da confiança e da exatidão destas informações. E a fim de suprir essa problemática surgiu o Projeto Acertar que busca prover orientações aos prestadores de serviços de abastecimento de água e esgotamento sanitário quanto às melhores práticas para gestão das informações do SNIS (BRASIL, 2018).

\section{As metas do PLANSAB}

A fim de estabelecer atingir as metas propostas no Plansab foram definidos indicadores que apresentariam os valores de referência dos parâmetros a serem monitorados. Originalmente, foram selecionados 23 indicadores, sendo 19 específicos dos quatro componentes do saneamento básico e outros 4 referentes a aspectos de gestão. Todavia, análises baseadas nos Relatórios de Avaliação Anual do Plano indicaram a necessidade de se alterar a fonte de dados e o conteúdo de alguns indicadores, bem como de realizar ajustes nos cálculos de outros.

Assim, a versão revisada de 2019 do Plano contém 29 indicadores, dos quais 24 são específicos para os quatro componentes do saneamento básico, sendo 8 relacionados ao abastecimento de água, 6 ao esgotamento sanitário, 8 ao manejo de resíduos sólidos e 2 à drenagem e manejo das águas pluviais urbanas (BRASIL, 2019).

Após as revisões do Plano houve necessidade de alterar alguns valores de indicadores e metas para o ano 2033. Logo, no horizonte de metas de médio prazo, o ano de 2023 , alguns indicadores também sofreram alterações desde o Plano original, contudo as mudanças não comprometeram a metodologia originalmente empregada (BRASIL, 2019).

A Tabela 1, abaixo, apresenta a situação dos serviços nos anos de 2010 e 2017 e as metas para 2023 
e 2033, em porcentagem, para o saneamento básico no Brasil e na região Norte, com base nos indicadores de abastecimento de água, esgotamento sanitário, coleta de resíduos sólidos e ocorrência de eventos hidrológicos em áreas urbanas.

Tabela 1: Metas específicas dos serviços públicos de saneamento básico no Brasil para os indicadores A1 e E1 (\%).

\begin{tabular}{|c|c|c|c|c|}
\hline Indicador & Fonte & Ano & Brasil & Norte \\
\hline \multirow{4}{*}{$\begin{array}{l}\text { A1. \% de domicílios urbanos e rurais abastecidos com água por rede de distribuição ou por } \\
\text { poço ou nascente }\end{array}$} & Censo & 2010 & 92,6 & 76,5 \\
\hline & Censo $^{1}$ & 2017 & 94,5 & 84,1 \\
\hline & Metas & 2023 & 96,1 & 90,7 \\
\hline & Plansab & 2033 & 99,0 & 94,0 \\
\hline \multirow{4}{*}{$\begin{array}{l}\text { E1. \% de domicílios urbanos e rurais servidos por rede coletora ou fossa séptica para os } \\
\text { excretas ou esgotos sanitários }\end{array}$} & Censo & 2010 & 67,0 & 33,5 \\
\hline & Censo $^{1}$ & 2017 & 73,6 & 35,9 \\
\hline & Metas & 2023 & 80,5 & 55,1 \\
\hline & Plansab & 2033 & 92,0 & 87,0 \\
\hline
\end{tabular}

${ }^{1}$ Valores obtidos a partir dos dados do Censo 2010, combinados com as variações anuais da PNAD.

Fonte: Adaptado de Brasil (2019).

O princípio comum quanto a universalização dos serviços básicos de saneamento aproxima as metas do Plano aos objetivos do ODS6. A universalização idealizada no Plansab (BRASIL, 2019) "remete à possibilidade de todos os brasileiros poderem alcançar uma ação ou serviço de que necessite, sem qualquer barreira de acessibilidade, seja legal, econômica, física ou cultura" e os objetivos da ONU para água e o saneamento são até 2030, alcançar o acesso universal e equitativo a água potável e segura para todos; e alcançar o acesso a saneamento e higiene adequados e equitativos para todos (UNITED NATIONS, 2015).

\section{METODOLOGIA}

\section{Caracterização do município em estudo}

O município de Porto Nacional tem uma área de $4.442,62 \mathrm{~km}^{2}$, com população estimada de 53.316 habitantes, sendo considerada a quarta maior cidade em aspectos populacionais do Tocantins (IBGE, 2020). O clima tem o clima C2WA'a", segundo o Método de Thornthwaite, com o clima úmido com poucas chuvas no inverno, tem evapotranspiração potencial média anual na faixa de $1.500 \mathrm{~mm}$, se dividindo no verão em 420 mm durante três meses consecutivos de temperaturas superiores (TOCANTINS, 2012).

O Ribeirão São João, que percorre o município, se configura a única fonte de abastecimento da cidade por meio de captação superficial. Com o acelerado crescimento populacional e o aumento do cultivo da soja e milho principalmente, aparece uma dúvida sobre o tratamento de suas águas para captação, abastecimento e como está o estado de sua fauna e flora (BALDUÍNO et al., 2019).

O período de concentração das precipitações ocorre entre os meses de outubro a abril, correspondendo a cerca de $80 \%$ da pluviosidade anual, enquanto o período seco corresponde aos meses de maio a setembro. $\mathrm{O}$ consumo de água é excessivo nesse período devido à alta temperatura, e o reservatório com baixo volume devido à estiagem (PORTO NACIONAL, 2018). 


\section{Seleção dos indicadores}

O presente estudo possui abordagem mista. Sendo parte da pesquisa qualitativa objetivando alcançar maior entendimento sobre os indicadores de cobertura de água e esgotamento sanitário e sobre a evolução dos serviços de saneamento em Porto Nacional. Nessa etapa foram consultadas base de dados locais, informações contidas na literatura e em documentos oficiais da Prefeitura Municipal, destacando-se o Plano Municipal de Água e Esgoto (PMAE) (PORTO NACIONAL, 2018).

Precedendo a coleta dos dados no SNIS efetuou-se a seleção dos indicadores do sistema referentes ao atendimento de água e esgotamento sanitário cuja abrangência é a população total do município. Concomitantemente a seleção dos indicadores foi realizada uma revisão acerca de suas estruturas de formulação. No que diz respeito ao serviço de atendimento de água, o indicador IN055 é a razão entre a população total atendida com abastecimento de água e a população total residente do município. Esse indicador é calculado por meio da Equação 1, abaixo:

$$
I N 055=\frac{A G 001}{G E 12 a} \times 100
$$

Onde AG001 é população total atendida com abastecimento de água e GE12a é população total residente do(s) município(s) com abastecimento de água, segundo o IBGE.

Quanto a cobertura total de coleta de esgotos sanitários, o indicador o IN056 é calculado por meio da Equação 2:

$$
I N 056=\frac{E S 001}{G E 12 a} \times 100
$$

Onde ES001 é população total atendida com esgotamento sanitário e GE12a é população total residente do(s) município(s) com abastecimento de água, segundo o IBGE.

\section{Coleta e análise de dados}

Os dados utilizados nesta etapa foram obtidos no sítio eletrônico do SNIS, referentes ao município de Porto Nacional, no período compreendido entre os anos de 2011 e 2019. Para identificar e analisar a evolução dos serviços no município, de Porto Nacional, foram selecionados e listados a seguir os parâmetros relacionados operacionais: 1) população total atendida com abastecimento de água; 2) população urbana atendida com abastecimento de água; 3) população total atendida com esgotamento sanitário; e 4) população urbana atendida com esgotamento sanitário.

Os dados utilizados na análise com relação a população atendida com água potável foram sistematizados a partir de 2011, os anos anteriores foram desconsiderados devido a existência de incompatibilidades de dados em relação às populações totais e atendidas. $E$ na análise dos dados de atendimento com esgotamento sanitário foram o período considerado foi 2011 a 2018, a partir dos dados fornecidos pelos prestadores no SNIS.

A etapa seguinte consistiu na comparação dos percentuais de prestação dos indicadores do ano de 2018 com o percentual de anos anteriores. A análise histórica de prestação foi desenvolvida separadamente por cada indicador selecionado. 


\section{RESULTADOS E DISCUSSÃO}

A Tabela 2 apresenta em cada um dos eixos analisados os valores da situação atual dos indicadores selecionados no SNIS frente as metas de médio (2023) e longo (2033) prazo previstas no Plansab.

Tabela 2: Situação atual dos indicadores de saneamento de Porto Nacional frente as metas do Plansab.

\begin{tabular}{lllllll}
\hline \multirow{2}{*}{ Eixo } & \multicolumn{2}{l}{$\begin{array}{l}\text { Situação atual dos } \\
\text { indicadores }\end{array}$} & \multicolumn{3}{l}{ Metas do Plansab } & \multicolumn{3}{c}{ Brasil } \\
\cline { 2 - 7 } & $\mathbf{2 0 1 8}$ & $\mathbf{2 0 1 8}$ & $\mathbf{2 0 2 3}$ & $\mathbf{2 0 3 3}$ & $\mathbf{2 0 2 3}$ & $\mathbf{2 0 3 3}$ \\
\hline Água (IN055) & $91,9 \%$ & $79 \%$ & $84 \%$ & $94 \%$ & $95 \%$ & $99 \%$ \\
Esgoto (IN056) & $72,9 \%$ & $52 \%$ & $63 \%$ & $87 \%$ & $81 \%$ & $92 \%$ \\
\hline
\end{tabular}

Os dados coletados no SNIS indicam que o município de Porto Nacional possui, em $2018,91,9 \%$ de atendimento total de água (IN055). Logo, o município ainda em 2018 conseguiu alcançar a meta de médio prazo prevista no Plansab para a região Norte do país em mais de 7 pontos percentuais (p.p.) e muito próximo de alcançar a meta nacional (95\%). No que diz respeito a cobertura de esgotamento sanitário (IN056), em 2018, o município possui 72,9\% das residências atendidas. Nesse quesito o município também alcançou já em 2018 a meta prevista para 2023 no Plansab e a aproximadamente 8 p.p. de atingir a meta para o Brasil. Apenas a nível de comparação o percentual de atendimento do Tocantins desse indicador para 2018 é de cerca de $26,4 \%$ de atendimento total.

A análise histórica do indicador de atendimento total de água de Porto Nacional (Figura 1), apresenta um cenário otimista no que diz respeito a universalização do serviço. Porto Nacional acompanhou a tendência do Tocantins e apresentou significativo aumento até 2015 e a partir de então manteve uma preocupante tendência decrescente nos 3 anos seguintes. Todavia evidencia-se a distância entre a prestação do município frente a do Estado e da região Norte.

Ao longo da análise de 2011 a 2018 Porto Nacional foi quem apresentou maior taxa de crescimento no atendimento de $1,17 \%$ superando o crescimento do estado do Tocantins $(0,99 \%)$ e da região norte (0,34\%). A Figura 2, a seguir, permite analisar a evolução do índice de atendimento total de esgoto em Porto Nacional entre os anos 2009 e 2018. A média de atendimento tocantinenses se manteve desde 2011 acima da regional e com taxa de crescimento de $8 \%$ para o período, superior à taxa de crescimento a do Norte do Brasil (1,11\%) e a pouco abaixo da estadual (8,76\%).

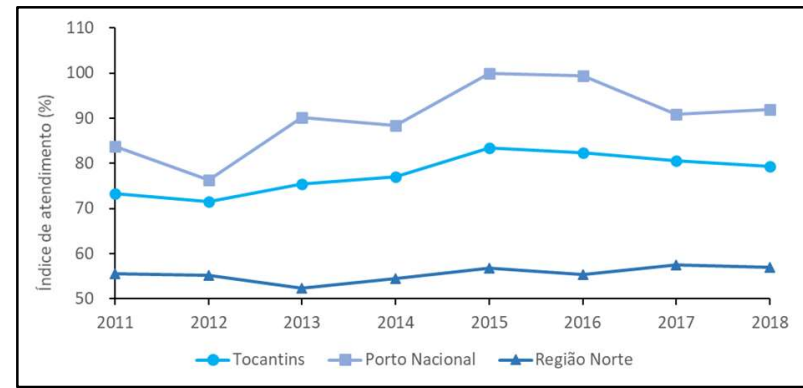

Figura 1: Evolução do índice de atendimento total de água (IN055) em Porto Nacional, no Tocantins e na região Norte, com base nos dados do SNIS dos anos de 2011 até 2018

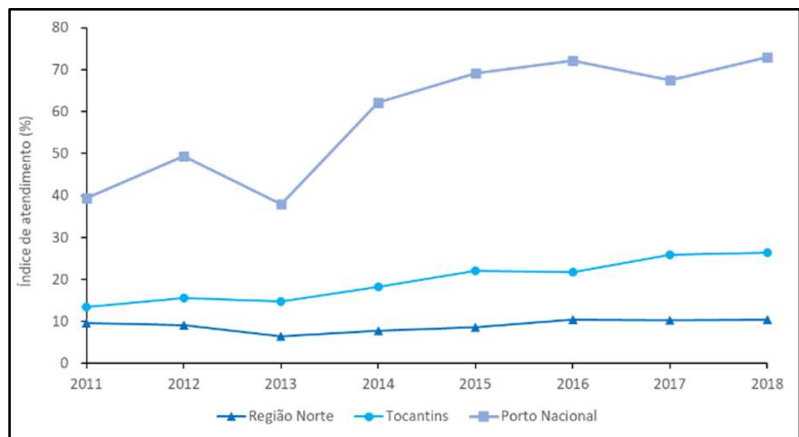

Figura 2: Evolução do índice de atendimento total de esgoto (IN056) em Porto Nacional, no Tocantins e na região Norte, com base nos dados do SNIS dos anos de 2011 até 2018 
A série histórica apresentou tendência linear positiva com coeficiente de determinação $\left(r^{2}\right)$ de 0,86. Esse valor de coeficiente evidencia um bom ajuste da série histórica do indicador IN056 ao modelo linear. Uma previsão simplista utilizando esse modelo aponta que, caso mantenha a tendência de atendimento, já em 2020 o município alcançará os $87 \%$ previstos como meta regional de longo prazo e a superar os $81 \%$ estipulados como meta de médio prazo (2023) para o país.

A realidade de Porto Nacional é bem diferente dos demais municípios do Estado no tocante ao esgotamento sanitário, os principais núcleos urbanos do município, já são atendidos, quase que integralmente, com sistema de esgotamento sanitário (PORTO NACIONAL, 2018).

\section{CONCLUSÕES}

O panorama de atendimento de água e esgoto sanitário em Porto Nacional é positivo, apesar de os índices de água apresentarem queda nos últimos anos. As metas de curto e médio prazo previstas no Plansab para a região Norte foram superadas e os indicadores se mantem próximos das metas previstas para o Brasil.

\section{REFERÊNCIAS}

ABES. Associação Brasileira de Engenharia Sanitária e Ambiental. Ranking ABES da universalização do saneamento. ABES, 2018.

BALDUINO, Â. R.. Análise dos impactos da agricultura na qualidade da água do reservatório de abastecimento de água no município de Porto Nacional - Tocantins. Tese (Doutorado em Ambiente) - Universidade Federal do Tocantins, Palmas, 2019.

BRASIL. Ministério das Cidades. Plano Nacional de Saneamento Básico. Brasília: Secretaria Nacional de Saneamento Ambiental, 2014

BRASIL. Ministério das Cidades. Diagnóstico dos Serviços de Água e Esgoto - 2018. Brasília: Sistema Nacional de Informações sobre Saneamento, 2018.

BRASIL. Ministério do Desenvolvimento Regional. Plano Nacional de Saneamento Básico. Brasil: Secretaria Nacional de Saneamento, 2019.
IBGE. Instituto Brasileiro de Geografia e Estatística. Cidades e Estados. Porto Nacional: IBGE, 2020.

PORTO NACIONAL. Plano Municipal de Água e Esgoto (PMAE). Porto Nacional, 2018.

SCRIPTORE, J. S.; TONETO JÚNIOR, R.. A estrutura de provisão dos serviços de saneamento básico no Brasil: uma análise comparativa do desempenho dos provedores públicos e privados. Revista de Administração Pública, v.46, n.6, p.1479-1504, 2012.

TOCANTINS. Secretaria do Planejamento. Superintendência de Planejamento e Gestão Central de Políticas Públicas: Diretoria Zoneamento Ecológico Econômico. Atlas do Tocantins: Subsídios ao Planejamento da Gestão Territorial. 5 ed. Palmas: SEPLAN, 2012.

UNITED NATIONS. Transforming our world: the 2030 Agenda for Sustainable Development. Resolution adopted by the General Assembly. Nova York: United Nations, 2015.

A CBPC - Companhia Brasileira de Produção Científica (CNPJ: 11.221.422/0001-03) detém os direitos materiais desta publicação. Os direitos referem-se à publicação do trabalho em qualquer parte do mundo, incluindo os direitos às renovações, expansões e disseminações da contribuição, bem como outros direitos subsidiários. Todos os trabalhos publicados eletronicamente poderão posteriormente ser publicados em coletâneas impressas sob coordenação da Sustenere Publishing, da Companhia Brasileira de Produção Científica e seus parceiros autorizados. Os (as) autores (as) preservam os direitos autorais, mas não têm permissão para a publicação da contribuição em outro meio, impresso ou digital, em português ou em tradução. 